\title{
Límites de edad para donante y receptor. Implicaciones del género: embarazo, anticoncepción, papiloma
}

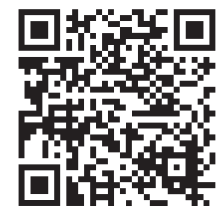

\author{
Age limits for kidney donor and transplant recipient. \\ Female sex implicactions: Pregnancy, contraception, papiloma virus
}

\author{
Roxana Villca-Gonzáles* \\ * Servicio de Nefrología. Hospital Regional "Lic. Adolfo López Mateos". ISSSTE
}

Edad del donador

\section{Limite inferior}

- Idealmente, los donadores deben ser mayores de 21 años.

- Los jóvenes menores de 18 años tienen contraindicación absoluta para donar.

- Los jóvenes entre 18-21 años tienen contraindicación relativa para donar: deben ser evaluados de manera cuidadosa sobre la comprensión del proceso de donación.

\section{Limite superior}

- No existe un límite de edad para la donación.

- La evaluación médica debe ser rigurosa para verificar que el donador tiene un estado de salud óptima y adecuada para la edad.

\section{Edad del receptor}

- La edad avanzada, por sí sola, no es una contraindicación para el trasplante renal.

- En este grupo debe enfatizarse la evaluación cardiovascular, gastrointestinal, neoplásica o infecciosa, capacidad cognitiva y fragilidad.

\section{EDAD DEL DONADOR}

El Foro de Ámsterdam y las Guías Británicas establecen que los menores de 18 años no deben ser considerados como donadores y establecen como contraindicación relativa la donación entre los 18-21 años. ${ }^{1,2}$

En general, la mayoría de las guías prohíben la donación en menores y algunas, excepcionalmente, permiten la donación, principalmente en gemelos idénticos, éste es un tema muy controvertido, puesto que se debe asegurar que el paciente tenga un desarroIlo psicosocial apto para decidir libremente, sin existir coerción. ${ }^{3}$

La necesidad de una mayor fuente de donadores ha hecho que mundialmente el número de donadores añosos se haya incrementado de manera importante en la última década. ${ }^{4}$ En este tipo de donadores es necesaria una evaluación exhaustiva para determinar su elegibilidad. Debe tenerse presente el deterioro de la función renal asociado al propio envejecimiento y descartar las comorbilidades frecuentes de la edad que alterarían la tolerancia al procedimiento quirúrgico.

En la evaluación de un registro de más de 80,000 donadores, no se encontró diferencia en la mortalidad quirúrgica perioperatoria en los mayores de 60 años comparados con donadores jóvenes. Aunque la mortalidad a largo plazo de los donadores añosos fue mayor 
en comparación con los donadores jóvenes, no se encontró diferencia en la supervivencia comparado con la población sana añosa no donadora. ${ }^{5}$ Otros reportes también han demostrado que no existe diferencia en cuanto a la incidencia de complicaciones postoperatorias comparado con otros grupos de edad. ${ }^{6,7}$

Otra preocupación en este grupo es el riesgo de desarrollar enfermedad renal crónica posterior a la donación, pero varios reportes muestran que este tipo de donadores presentan una disminución de la tasa de filtrado glomerular (TFG) en el seguimiento (alrededor de $30-40 \%$ del valor predonación hasta los 3-6 años) similar a la observada en donadores jóvenes. ${ }^{8,9}$ Por lo tanto, la donación se considera segura. ${ }^{10}$

La mayoría de las guías no establecen un límite de edad per se para la donación y no existe evidencia que contraindique la donación sobre los 60 o 70 años. ${ }^{11}$ Una de las preocupaciones en este tipo de donador radica en la supervivencia del injerto donado. Se ha demostrado que los injertos de donadores $>60$ años tienen una supervivencia similar o mejor a la de injertos de donadores cadavéricos de criterios estándar y una supervivencia mayor que los injertos de donadores cadavéricos de criterios extendidos. ${ }^{12}$

Asimismo, otros reportes muestran resultados de donadores añosos muy satisfactorios. Para receptores de injertos de donadores $>60$ años, la supervivencia a cinco años está entre $80-100 \%$ y la supervivencia del injerto censurada para muerte entre $95-100 \%$, resultados que fueron similares si el injerto provenía de un donador joven. ${ }^{13-16}$

\section{EDAD DEL RECEPTOR}

Queda claro que la esperanza de vida se ha incrementado y por ende el número de pacientes adultos mayores con enfermedad renal crónica terminal (ERCT). Todo paciente con ERCT es candidato a ser evaluado para el protocolo de trasplante renal, sin importar la edad. Por todo lo anterior, el número de receptores mayores de 65 años aceptados para trasplante se ha incrementado en la mayor parte de los centros de trasplantes. ${ }^{17}$

Aunque existen características especiales relacionadas con el receptor geriátrico de trasplante renal que deben considerarse de manera cuidadosa, como el daño cognitivo y la fragilidad, existe evidencia que muestra que pacientes adultos mayores (incluso de más de 70 años) se benefician en supervivencia y mejoría en la calidad de vida con el trasplante comparado a permanecer en diálisis. ${ }^{18-20}$

Es importante señalar que este grupo de pacientes tiene una baja supervivencia si permanecen en lista de espera (46\% de los pacientes mayores de 60 años en lista de espera fallecen antes de recibir un trasplante), ${ }^{21}$ por lo que es importante un trasplante temprano utilizando injertos provenientes de donadores fallecidos de criterios extendidos ${ }^{22}$ o donadores vivos añosos como ya mencionamos previamente.

Es importante seleccionar de forma apropiada al receptor añoso, enfatizando en la evaluación cardiovascular, gastrointestinal, neoplásica, infecciosa, capacidad cognitiva y fragilidad, puesto que «muerte con injerto funcional» es la principal causa de pérdida del injerto en este grupo de edad..$^{23,24}$

\section{IMPLICACIONES DEL SEXO}

\section{Donador femenino}

- Las mujeres en edad fértil no deben ser descartadas como potenciales donadores.

- Debe discutirse planes de embarazo en toda mujer candidata a donadora, así como conocer complicaciones en embarazos previos.

- Es contraindicación absoluta la donación durante el curso de un embarazo.

- Se debe realizar prueba de embarazo previo a la cirugía en mujeres en edad fértil.

- En el caso de embarazo previo a la donación es recomendable posponer la donación por un año después del nacimiento de acuerdo con las necesidades del bebé y de la madre.

- En el caso de embarazo posterior a la donación: existe evidencia que sugiere que la donación podría incrementar el riesgo de hipertensión gestacional y preeclampsia.

En la evaluación de potenciales donadoras es fundamental conocer antecedentes y características de embarazos previos, así como planes de futuros embarazos. Es importante señalar que es contraindicación absoluta la donación durante el curso de un embarazo, por lo que se sugiere realizar la prueba de embarazo previo al inicio del protocolo de estudio y previo a la cirugía en mujeres en edad fértil. ${ }^{25}$

Los antecedentes de complicaciones en embarazos previos son importantes, puesto que posterior a un embarazo con preeclampsia existe un mayor riesgo de albuminuria, ${ }^{26}$ así como mayor riesgo de desarrollar ERCT; ${ }^{27}$ por otro lado, el desarrollar diabetes gestacional también está asociada con ERCT. ${ }^{28}$

En el caso de embarazo previo a la donación es recomendable posponer la donación por un año des- 
pués del nacimiento de acuerdo con las necesidades del bebé y de la madre. ${ }^{25}$

La literatura actual sugiere que las complicaciones en el embarazo son más comunes en los embarazos postdonación que en embarazos predonación.

Un reporte de 1,589 mujeres donadoras mostró que los embarazos postdonación (versus embarazos predonación) se asociaron a una menor probabilidad de embarazo a término (73.7 vs $84.6 \%$ ), mayor riesgo de pérdida fetal (19.2 vs $11.3 \%$ ), mayor riesgo de hipertensión gestacional (5.7 vs $0.6 \%$ ), proteinuria (4.3 vs $1.1 \%$ ) y preeclampsia (5.5 vs $0.8 \%$ ). Si bien las complicaciones de los embarazos postdonación son similares a las reportadas en la población general son mayores a la de los embarazos predonación. ${ }^{29}$

Otro reporte evaluó a 85 donadoras que se compararon con 510 mujeres sanas no donadoras con similares condiciones de salud. Se encontró una mayor frecuencia de preeclampsia en las donadoras (11\%) en comparación con las no donadoras (5\%). Cabe señalar que en dicho estudio no encontraron diferencias en las tasas de nacimiento pretérmino o bajo peso al nacer. ${ }^{30}$ Recientemente otro reporte no demostró diferencias en cuanto a frecuencia de preeclampsia ni de hipertensión gestacional, ni desenlaces fetales adversos entre embarazos postdonación y embarazos en no donadoras. $^{31}$

Por lo anterior, toda paciente con deseos de embarazo posterior a la donación debe conocer que puede tener un mayor riesgo de preeclampsia o hipertensión gestacional. Asimismo, se deberá brindar una asesoría para modificar factores de riesgo asociados con patologías hipertensivas en el embarazo.

\section{REFERENCIAS}

\section{Límites de edad: donador-receptor}

1. Delmonico F, Council of the Transplantation Society. A report of the Amsterdam forum on the care of the live kidney donor: data and medical guidelines. Transplantation. 2005; 79: S53-S66.

2. British Transplantation Society/Renal Association. United Kingdom Guidelines for Living Donor Kidney Transplantation. 4th edition. 2018.

3. Thys K, Van Assche K, Nobile $\mathrm{H}$ et al. Could minors be living kidney donors? A systematic review of guidelines, position papers and reports. Transplant Int. 2013; 26: 949-960.

4. Hart A, Smith JM, Skeans MA, Gustafson SK, Wilk AR, Castro S et al. OPTN/SRTR 2017 Annual Data Report: Kidney. Am J Transplant. 2019; 19: 19-123.

5. Segev DL, Muzaale AD, Caffo BS, Mehta SH, Singer AL, Taranto SE et al. Perioperative mortality and long-term survival following live kidney donation. JAMA. 2010; 303: 959-966.
6. O'Brien B, Mastoridis S, Sabharwal A, Hakim N, Taube D, Papalois $V$ et al. Expanding the donor pool: living donor nephrectomy in the elderly and the overweight. Transplantation. 2012; 93: 1158-1165.

7. Jacobs SC, Ramey JR, Sklar GN, Bartlett ST. Laparoscopic kidney donation from patients older than 60 years. J Am Coll Surg. 2004; 198: 892-897.

8. Toyoda M, Yamanaga S, Kawabata C et al. Long-term safety of living kidney donors aged 60 and older. Transplant Proc. 2014; 46: 318-320.

9. Saito $\mathrm{T}$, Uchida $\mathrm{K}$, Ishida $\mathrm{H}$ et al. Changes in glomerular filtration rate after donation in living kidney donors: a single-center cohort study. Int Urol Nephrol. 2015; 47: 397-403.

10. Dols LFC, Kok NFM, Roodnat JI et al. Living kidney donors: impact of age on long-term safety. Am J Transplant. 2011; 11: 737-742.

11. Berger JC, Muzaale AD, James N, Hoque M, Wang JM, Montgomery RA et al. Living kidney donors ages 70 and older: recipient and donor outcomes. Clin J Am Soc Nephrol. 2011; 6: 2887-2893.

12. Englum BR, Schechter MA, Irish WD, Ravindra KV, Vikraman DS, Sanoff SL et al. Outcomes in kidney transplant recipients from older living donors. Transplantation. 2015; 99: 309e15.

13. Gallinat A, Paul A, Treckmann JW et al. Single-center experience with live kidney donors 60 years of age or older. Am Surg. 2014; 80: $1230-1236$.

14. Patel HV, Kute VB, Shah PR et al. Outcome of renal transplantation from older living donors compared to younger living donor in developing country. Ren Fail. 2014; 36: 15161519.

15. Lionaki S, Kapsia H, Makropoulos I et al. Kidney transplantation outcomes from expanded criteria donors, standard criteria donors or living donors older than 60 years. Ren Fail. 2014; 36: 526-533.

16. Li Y, Li J, Fu Q et al. Kidney transplantation from living related donors aged more than 60 years: a single center experience. Ren Fail. 2013; 35: 1251-1254.

17. Schaeffner ES, Rose C, Gill JS. Access to kidney transplantation among the elderly in the United States: a glass half full, not half empty. Clin J Am Soc Nephrol. 2010; 5 (11): 2109-2114.

18. Wolfe RA, Ashby VB, Milford EL, Ojo AO, Ettenger RE, Agodoa LY et al. Comparison of mortality in all patients on dialysis, patients on dialysis awaiting transplantation, and recipients of a first cadaveric transplant. N Engl J Med. 1999; 341: 1725-1730.

19. Knoll GA. Kidney transplantation in the older adult. Am J Kidney Dis. 2013; 61: 790-797.

20. Rao PS, Merion RM, Ashby VB et al. Renal transplantation in elderly patients older than 70 years of age: results from the Scientific Registry of Transplant Recipients. Transplantation. 2007; 83: 1069-1074.

21. Schold JD, Srinivas TR, Sehgal AR, Meier-Kriesche HU. Half of kidney transplant candidates who are older than 60 years now placed on the waiting list will die before receiving a deceased-donor transplant. Clin J Am Soc Nephrol. 2009; 4: 1239-1245.

22. Ojo AO, Hanson JA, Meier-Kriesche H, Okechukwu CN, Wolfe $R A$, Leichtman $A B$ et al. Survival in recipients of marginal cadaveric donor kidneys compared with other recipients and wait-listed transplant candidates. J Am Soc Nephrol. 2001; 12 : 589-597.

23. Boesmueller C, Biebl M, Scheidl S, Oellinger R, Margreiter $\mathrm{C}$, Pratschke $\mathrm{J}$ et al. Long-term outcome in kidney transplant recipients over 70 years in the Eurotransplant Senior Kidney 
Transplant Program: a single center experience. Transplantation. 2011; 92: 210-216.

24. Sener A, Schweitzer EJ, Munivenkatappa R, Cooper M, Bartlett ST, Philosophe B et al. Deceased-donor renal transplantation in the geriatric population demonstrates equal graft survival compared with younger recipients. Transplantation. 2009; 87: 1549-1554.

\section{Implicaciones de género}

25. Lentine KL, Kasiske BL, Levey AS et al. Kidney Disease: Improving Global Outcomes (KDIGO) Living Kidney Donor Work Group. KDIGO Clinical Practice Guideline on the Evaluation and Care of Living Kidney Donors. Transplantation. 2017; 101 (Suppl 8S): S1-S109.

26. McDonald SD, Han Z, Walsh MW et al. Kidney disease after preeclampsia: a systematic review and meta-analysis. Am J Kidney Dis. 2010; 55: 1026-1039.

27. Vikse BE, Irgens LM, Leivestad T et al. Preeclampsia and the risk of end- stage renal disease. N Engl J Med. 2008; 359: 800-809.
28. Beharier O, Shoham-Vardi I, Pariente G et al. Gestational diabetes mellitus is a significant risk factor for long-term maternal renal disease. J Clin Endocrinol Metab. 2015; 100: 1412-1416.

29. Ibrahim HN, Akkina SK, Leister E, Gillingham K, Cordner G, Guo $\mathrm{H}$ et al. Pregnancy outcomes after kidney donation. Am J Transplant. 2009; 9: 825-834.

30. Garg AX, Nevis IF, McArthur E, Sontrop JM, Koval JJ, Lam NN et al, and for the DONOR Network. Gestational hyper- tension and preeclampsia in living kidney donors. N Engl J Med. 2015; 372: 124-133.

31. Yoo KD, Lee H, Kim Y, Park S, Park JS, Hong JS et al. Maternal and fetal outcomes of pregnancies in kidney donors: A 30-year comparative analysis of matched non-donors in a single center. Kidney Res Clin Pract. 2018; 37 (4): 356-365.

Correspondencia:

Dra. Roxana Villca-Gonzáles

E-mail: roxanavillca@yahoo.com 